\title{
FRECUENCIA EN LA PRESCRIPCIÓN DE MEDICAMENTOS EN GESTANTES ANÉMICAS QUE ACUDAN AL CENTRO DE SALUD CIUDAD NUEVA. OCTUBRE 2001-SEPTIEMBRE 2002
}

\author{
José Rómulo Calle Munárriz' ; Edgard Guido Calderón Copa ${ }^{2}$
}

\begin{abstract}
RESUMEN
La investigación es Básico. Descriptivo con diseño no Experimental y Prospectivo, se realizó en 399 gestantes, de las cuales se tomó como muestra representativa, 170 pacientes.

Con relación a la Frecuencia a la Prescripción de medicamentos a gestantes anémicas, se puede decir que el $40.59 \%$ de anémicas fueron prescritas tanto con fármacos antianémicos como otro tipo de fármacos para patologias como Infección Urinaria. En el caso de fármacos diferentes a los antianémicos, resultan ser $16.47 \%$, los medicamentos antianémicos se presentan en un $18.24 \%$. El $59.41 \%$ de gestantes anémicas y no anémicas no fueron prescritas. El $78.24 \%$ son gestantes con anemia, de las cuales en un menor valor fueron prescritas con Sulfato Ferroso.
\end{abstract}

Respecto a la Frecuencia en la Prescripción de Medicamentos Antianémicos por trimestre de gestación, se encontró que el tercer trimestre con $48.38 \%$ es el más prescrito. Sobre la Frecuencia en la Prescripción de Medicamentos Antianémicos de acuerdo al tipo de gestantes, se encontró que hay mayor prescripción en Multigestas (58.06\%) que las Primigestas (41.94\%).

\section{A B S TR A C T}

The investigation is Basic, Descriptive with design not Experimental and Prospective, one carries out in 399 pregnants, of which he/she took like representative sample, 170 patients.

With relationship to the Frequency to the Prescription of medications to anemic pregnants, one can say that $40.59 \%$ of anemic was prescribed so much with medications antianemics as another medications type for pathologies like Infection Urinary. In the case of medications different to the antianemics, they turn out to be $16.47 \%$, the medications antianemics are presented in $18.24 \% .59 .41 \%$ of anemic and not anemic pregnants was not prescribed. $78.24 \%$ is pregnancy with anemia, of which were prescribed with Ferrous Sulfate in a smaller value.

Regarding the Frequency in the Prescription of Medications Antianemics for gestation trimester, was found that the third trimester with $48.38 \%$ is the most prescribed one. About the Frequency in the Prescription of Medications Antianemics according to the pregnants type, was found that there is bigger prescription in Multigestas (58.06\%) that the Primigestas (41.94\%).

\section{INTRODUCCIÓN}

La terapia farmacológica durante el embarazo constituye un problema de importancia en la práctica médica general cuando se administra medicamentos a la madre, el nuevo ser en desarrollo puede llegar a se involuntariamente afectado. Por resultado de la comprensible adopción de una actitud conservadora en estas situaciones, en el sentido de restringir el uso de medicamentos, siempre y cuando no se llegue a decisiones extremas, como prescribir su administración en casos debidamente justificados.

La Anemia es un problema serio en Salud Públicá, ya que atecta al $30 \%$ de la población mundial siendo esta, la reducción del volumen total de los glóbulos rojos en el Hematocrito o la disminución de la concentración de Hemoglobina por debajo del índice normal. Entre las causas más importantes de anemia podemos mencionar que pueden ser por pérdida y destrucción de hematies y la ocasionada por e desorden intrínseco de la médula ósea. Las anemias carenciales o por deficiencia nutricional comprender a las anemias producidas por déficit de los nutrientes necesarios para la eritropoyesis. La más frecuente e la anemia ferropénica y la megaloblástica. 
Todo medicamento tiene riesgos: "Ninguna sustancia química puede ser creada y administrarse a hombres y mujeres, por via oral, nasal, retinal, cutánea, subcutánea, rectal o vaginal, sin estar totalmente libre de riesgo. Lo que convierte a un medicamento en un problema, no tanto por los riesgos farmacológicos inherentes, sino el modo en que es prescrito y usado.

Como el Centro de Salud Ciudad Nueva, no se han realizado estudios sobre la Frecuencia en la Prescripción de Medicamentos a Gestantes Anémicas, nos motivo a realizar la presente investigación con la seguridad de que los resultados que se vayan a encontrar servirán al establecimiento de Salud para la toma de decisiones.

\section{PLANTEAMIENTO DE LA INVESTIGACIÓN}

\section{DESCRIPCIÓN DEL PROBLEMA}

La mayoria de gestantes tienen bajos niveles de hierro en la sangre, debido al proceso mismo, porque el volumen de la sangre aumenta para abastecer al niño en crecimiento, a pesar que el ser humano tiene grandes reservas de hierro en el hígado y la médula ósea, que se usan durante el embarazo (3). En el Distrito de Ciudad Nueva los recursos económicos de las familias son escasos esto por el período de recesión que se vive en el pals. Razón por la cuál la población de este distrito descuida la alimentación nutritiva lo cual se da de manera más agravada en las gestantes.

\section{ANTECEDENTES DEL PROBLEMA}

- La estimación de la prevalencia de la deficiencia de hierro en Estados Unidos y otros paises desarrollados depende de la situación económica de la población estudiada. En países subdesarrollados del 20 al $40 \%$ de los lactantes y gestantes pueden estar afectados; mientras que en Estados Unidos y Suecia hay estudios que indican que la prevalencia en estos paises es de 5 a $10 \%$ según Hallberg y Colaboradores, en 1979 (4).

- Anon en 1989 sugirió que todas las gestantes de países en desarrollo deben recibir suplementos de hierro durante los últimos cuatro a cinco meses del embarazo (3).

- Llosa L. en 1993, reportó que en el Hospital de la Maternidad de Lima, la frecuencia de anemia en gestante varia de $47 \%$ al $86 \%$ (5).

- Calle J., Calderón E., en un estudio realizado en Ciudad Nueva (Tacna-Perú), el año 2001 reportaron que el $22,22 \%$ de gestantes tomaron Sulfato Ferroso en tabletas e infirieron de acuerdo al número de veces de prescripción este medicamento que el $21,57 \%$ de gestantes presentaron un cuadro anémico, dato que no necesariamente evidencia la frecuencia de anemias (2).

\section{MATERIAL Y MÉTODO DISEÑO DE INVESTIGACIÓN.}

Este Trabajo de Investigación es Básico Descriptivo con un Diseño no Experimentaly Prospectivo que se realizó en gestantes que acudieron al C.S. Ciudad Nueva de Octubre 2001 a Septiembre 2002, con una población de 399 gestantes, de las cuales se tomó como muestra representativa a 170 pacientes.

\section{MUESTRAS DE ESTUDIO}

La muestra de estudio es no probabilística, se tomó 170 gestantes, que representaron el $42.61 \%$ del universo que acudieron al Centro de salud Ciudad Nueva durante el tiempo señalado.

\section{INDICADORES}

$\S$ Frecuencia de medicamentos prescritos.

$\S$ Trimestre de gestación.

$\S$ Número de partos.

$\S$ Número de gestaciones.

$\S$ Edad.

$\S$ Grado de instrucción.

$\S$ Estado civil.

$\S$ Hemoglobina.

$\S$ Hematocrito.

$\S$ Otras enfermedades

\section{RESULTADOS}

TABLA N $N^{\circ} 01$

GESTANTESANEMMICASQUERECIBIERONPRESCRIPCIÓN

\begin{tabular}{|c|c|c|}
\hline & & \\
\hline & & \\
\hline C/Prescripción & 69 & 40.59 \\
\hline S/Prescripción & 101 & 59.41 \\
\hline Total & 170 & 100.00 \\
\hline
\end{tabular}

TABLA N 02

PRESCRIPCIÓNDE OTROS MEDICAMENTOSAGESTANTES ANÉMICAS

\begin{tabular}{|c|c|c|}
\hline & & \\
\hline C/Prescripción & 28 & 16.47 \\
\hline S/Prescripción & 142 & 83.53 \\
\hline TOTAL & 170 & 100.00 \\
\hline
\end{tabular}


TABLA N ${ }^{\circ} 03$

PRESCRIPCIÓNDE FARMACOSANTIANÉMICOS AGESTANTES ANÉMICAS

\begin{tabular}{|c|c|c|}
\hline & & \\
\hline Antianémicos & 31 & 18.24 \\
\hline Otros & 139 & 81.76 \\
\hline TOTAL & 170 & 100.00 \\
\hline
\end{tabular}

TABLA N ${ }^{\circ} 04$

PRESCRIPCIÓNDE OTROS FARMACOSAGESTANTES SIN ANEMIA

\begin{tabular}{|c|c|c|c|}
\hline & & & \\
\hline C/Prescripción & 10 & 27.03 & 5.88 \\
\hline S/ Prescripción & 27 & 72.97 & 94.21 \\
\hline TOTAL & 37 & 100.00 & 100.00 \\
\hline
\end{tabular}

TABLA N ${ }^{\circ} 05$

FRECUENCIADEANEMIAS EN GESTANTES

\begin{tabular}{|c|c|c|}
\hline & & \\
\hline ClAnemla & 133 & 78.24 \\
\hline S/Anemia & 37 & 21.76 \\
\hline TOTAL & 170 & 100.00 \\
\hline
\end{tabular}

TABLA N ${ }^{\circ} 06$

HEMOGLOBINAVERSUS GESTANTES

\begin{tabular}{|c|c|c|}
\hline & & \\
\hline $8.00-8.99$ & 2 & 1.18 \\
\hline $9.00-9.99$ & 7 & 4.12 \\
\hline $10.00-10.99$ & 65 & 38.23 \\
\hline $11.00-11.99$ & 59 & 34.71 \\
\hline $12.00-12.99$ & 20 & 11.76 \\
\hline $13.00-13.99$ & 11 & 6.47 \\
\hline $14.00-16.99$ & 6 & 3.53 \\
\hline TOTAL & 170 & 100.00 \\
\hline
\end{tabular}

TABLA N ${ }^{\circ} 07$

GESTANTESANÉMICAS CONYSINPRESCRIPCIÓN

\begin{tabular}{|c|c|c|c|c|c|c|c|c|}
\hline CIANEMA & 59 & 34.71 & 85.51 & 74 & 43.53 & 75.15 & 133 & 78.24 \\
\hline SIANEMA & 10 & 5.88 & 14.49 & 27 & 15.88 & 28.85 & 37 & 21.76 \\
\hline TOTAL & 69 & 40.50 & 100.00 & 101 & 59.41 & 100.00 & 170 & 100.00 \\
\hline
\end{tabular}

TABLA No 08

PRESCRIPCIÓNDEMEDICAMENTOSPORTRIMESTRE

\begin{tabular}{|c|c|c|c|}
\hline & \multicolumn{3}{|c}{} \\
\hline PRIMERO & 8 & 25.81 & 6.02 \\
\hline SEGUNDO & 8 & 25.81 & 6.02 \\
\hline TERCERO & 15 & 48.38 & 11.28 \\
\hline TOTAL & 31 & 100.00 & 23.32 \\
\hline
\end{tabular}

TABLA N ${ }^{\circ} 09$

PRESCRIPCIÓNDEANTIANÉMICOS VERSUSTIPODE GESTANTES

\begin{tabular}{|c|c|c|c|}
\hline & & & \\
\hline & & & \\
\hline & & & \\
\hline PRIMIGESTA & 13 & 41.94 & 9.78 \\
\hline MLILTIGESTA & 18 & 58.06 & 13.54 \\
\hline TOTAL & 31 & 100.00 & 23.32 \\
\hline
\end{tabular}

TABLA N ${ }^{\circ} 10$

PRESCRIPCIÓNDEANTIANÉMICOSAGESTANTESDE ACUERDO AL GRUPO ETAREO

\begin{tabular}{|c|c|c|c|}
\hline & & & \\
\hline $16-10$ ANOS & 7 & 2258 & 5.28 \\
\hline $20-24$ AIIOS & 7 & 22.58 & 5.26 \\
\hline $25-29$ ANOS & 10 & 32.26 & 7.52 \\
\hline 30-34 ANOS & 4 & 12.90 & 3.02 \\
\hline MAS DE 35 ANOS & 3 & 9.68 & 2.26 \\
\hline TOTAL & 31 & 100.00 & 23.32 \\
\hline
\end{tabular}

\section{DISCUSIÓN}

El presente trabajo de Investigación determina la Frecuencia en la Prescripción de Medicamentos en gestantes anémicas que acudieron de Octubre del 2001 hasta Septiembre del 2002 al Centro de Salud Ciudad Nueva.

Las vitaminas y minerales, especialmente el hierro, son regularmente prescritas durante el embarazo. Frecuentemente se toman con la creencia que son sustancias naturales y que por lo tanto no son dañinas. Sin embargo, esto no es asi, la sobredosis de hierro puede ser extremadamente peligrosa y conducir a una insuficiencia cardiaca.

Administrar suplementos de hierro que correspondan a un enfoque curativo son más importantes los esfuerzos para mejorar la nutrición en general y asegura que las personas tengan acceso a los alimentos que reúnan todas las necesidades nutricionales (3).

Con relación a la Frecuencia a la Prescripción de medicamentos a gestantes anémicas (Tabla $\mathbf{N}^{0} 10$ ), se puede decir que el $40,59 \%$ de anémicas fueron 
prescritas tanto fármacos antianémicos como otro tipo de fármacos para patologlas como Infección Urinaria (8) que se debe a la disminución del tono y peristalsis ureterales, y flujo vaginal debido a la vida sexual activa, pudiendo modificar el medio ácido y los bacilos de Doderlein de la vagina (3). En el caso de fármacos diferentes a los antianémicos, resultan ser $16,47 \%$ (Tabla $\mathrm{N}^{\circ}$ 02), los medicamentos antianémicos se presentan en un $18,24 \%$ (Tabla N ${ }^{\circ} 03$ ), valor menor al propuesto en la Hipótesis $(20 \%)$, siendo bajo teniendo en cuenta que la frecuencia de anemias en gestantes es $78,24 \%$ (Tabla $N^{\circ} 05$ ). La explicación es que hay un gran porcentaje de anémicas que tienen una hemoglobina de 10.00 a 11.99 , constituyendo un $72,94 \%$, a una gran parte de este grupo no se les prescribe Sulfato Ferroso, hasta que se presente probablemente mayor deficiencia, cuando después de evaluarla el facultativo, lo determine.

Los valores de referencia de hemoglobina $(\mathrm{Hb})$ se ha tomado de la Tabla modificada de Little y colaboradores del año 1997, para poder determinar quienes presentan anemia, en esta se establecen como valores normales, en mujeres de 12 a 18 años y en adultas el valor de $\mathrm{Hb}$ de 12,00 a 16,00 (3). Se puede mencionar también que más del $50 \%$ de anemias son debido a causas de tipo nutricional, por falta de aporte de hierro en la dieta (3). El valor de $78,24 \%$, encontrado en nuestro estudio, esta en el rango del valor encontrado en otro realizado por el Instituto de Investigación Nutricional de la Molina, en el Hospital de Maternidad de Lima que fue de $47 \%$ al $86 \%$ de gestantes anémicas.(5).

En la Tabla № 7 , se puede observar que el $34,71 \%$ de gestantes son prescritas con fármacos antianémicos y otros fármacos, el $5,88 \%$ de gestantes no anémicas fueron prescritas con otro tipo de fármacos, el $59,41 \%$ de gestantes anémicas y no anémicas no fueron prescritas. También se observa que el $78,24 \%$ son gestantes con anemia, de las cuales en menor valor fueron prescritas con Sulfato Ferroso, probablemente se deba a que su utilización en la prescripción moderada sea por los efectos colaterales que puede causar y por que no hay una anemia severa.

La tabla $N^{\circ} 8$, nos da la información sobre la frecuencia en la prescripción de medicamentos antianémicos por trimestre de gestación, encontrándose que el tercer trimestre $(48,38 \%)$ es el más prescrito, coincidiendo con las recomendaciones que se hacian respecto a que trimestre es recomendable prescribirlo (3). En este trimestre hay mayor deficiencia nutricional de hierro.

En la tabla $N^{\circ} 9$, sobre la frecuencia de la prescripción de medicamentos antianémicos de acuerdo al tipo de gestantes, se encontró que hay mayor prescripción en las multigestas $(58,06 \%)$ y menor en las primigestas $(41,94 \%)$, concluyéndose que presentar inayor anemia las multigestas que probablemente se deba a los repetidos embarazos.

En la tabla $\mathrm{N}^{\circ} 10$, se observa que grupo etáreo presenta mayor frecuencia, siendo el de 25 a 29 años con $32,26 \%$, seguido por los grupos de 15 a 19 y 20 a 24 años de edad ambos con $22,58 \%$. probablemente se debe a que en el Distrito Ciudad Nueva las mujeres de esta edad descuidan la alimentación nutricional por ende de hierro, por la situación económica difícil que pasa el país y se ven obligados a trabajar para ayudar al sostenimiento diario del hogar. A partir de los 30 años disminuye debido a que son menos las gestantes de este grupo.

En general hay un alto porcentaje de anemia $78,24 \%$ por los argumentos anteriormente mencionados, también se puede destacar la poca difusión que hay sobre qué alimentos contienen el micronutriente hierro importante durante el embarazo. El embarazo es un proceso natural, en donive el volumen de sangre en las mujeres aumenta para abastecer al feto en desarrollo y más aún durante la deficiencia por falta de una alimentación nutritiva adecuada de hierro (3).

\section{CONCLUSIONES}

Del presente estudio sobre gestantes anémicas encontradas en el Centro de Salud Ciudad Nueva podemos llegar a las siguientes conclusiones:

Con referencia a la Frecuencia en la Prescripción de Medicamentos a Gestantes anémicas:

- El $40,59 \%$ de gestantes fueron prescritas.

- El $59,41 \%$ no fueron prescritas.

Con respecto a la Frecuencia en la Prescripción de Fármacos díferente a los Antianémicas:

- El 16,47\% representan a gestantes anémicas que fueron prescritas con fármacos diferentes a los antianémicos.

- El $83,53 \%$ no fueron prescritas con fármacos diferentes a los antianémicos.

En referencia a la Frecuencia en la Prescripción de Fármacos Antianémicos:

- El $18,24 \%$ de 170 gestantes representa a las que se les prescribió fármacos antianémicos.

- El $81,76 \%$ representa a las gestantes no prescritas con antianémicos.

\section{Referente a la Frecuencia de Anemias en} Gestantes:

- El $78,24 \%$ de gestantes tienen anemia.

- El $21,76 \%$ de gestantes no tienen anemia 


\section{Referente a la Hemoglobina en Gestantes Anémicas:}

- El $34,71 \%$ de gestantes anémicas pertenecen al grupo de 11,00 a 11,99 de hemoglobina.

- El $38,23 \%$ pertenece al grupo de 10,00 a 10,99 .

- El $11,76 \%$ representa al grupo de 12,00 a 12,99 principalmente.

De la Frecuencia de Fármacos Antianémicos de acuerdo al Trimestre se puede concluir:

- Que el $25,81 \%$ representa al primer y segundo trimestre, con el mismo valor.

- Que el $48,38 \%$, representa al tercer trimestre.

\section{Con referencias al Tipo de Gestante:}

- Las multigestas representa a las gestantes más frecuentemente prescritas con antianémicos, en un $58,06 \%$.

- Las primigestas, en un $41,94 \%$.

Finalmente, con referencia al grupo etáreo de gestantes más frecuentemente prescritas con antianémicos:

- El grupo de 25 a 29 años de edad es el más frecuentemente prescrito, con un $32,26 \%$.

- El de 15 a 19 años y 20 a 24 años, representan un $22,58 \%$, separadamente.

- El de menor frecuencia en la prescripoión es el grupo de más de 35 años con $9,68 \%$.

\section{REFERENCIA BIBLIOGRÁFICA}

1. ANEMIA. "La Prescripción". Noviembre 1994. Publicación de la UNICEF para Prescriptores.

2. CALLE, CALDERÓN, FIGUEROA. "Frecuencia en el Uso de Medicamentos por Las Gestantes que Acuden al Centro de Salud Ciudad Nueva en el Año 2000". Revista Médica Basadrina. Vol. 1: 14 -17. Tacna - Perú. 2001.

3. CHETLEY, Andrew. "Medicamentos Problema". Segunda Edición. Estados Unidos. 1995.

4. GOODMAN, Alfred. "Las Bases Farmacologicas de la Terapéutica". Séptima Edición. Edit. PANAMERICANA S.A. Argentina 1987.
5. LLOSA, L. “Anemias Nutricionales. Separata para Capacitación de Profesionales". Lima. Servicio de Medicinas Próvida. 1993.

6. LLOSA, L. "Carencia de Hierro y Anemia Ferropénica". Medicamentos y Salud Popular.

7. Organización Panamericana de la Salud. Grupo Técnico de Anemia. en MuJeres Gestantes. OPS. 1993. (Boletín $N^{\circ} 1$. Diciembre 1993).

8. SERVICIO DE MEDICINAPROVIDA. "Manual de Terapéutica Médica". Lima - Perú 1998. 